\title{
Analytical investigation of thermal radiation effect on nanofluid forced convection in a semi porous channel
}

\author{
Sahar Moazami Goudarzi ${ }^{1}$ and D.D. Ganji ${ }^{2}$ \\ ${ }^{1}$ Department of Mechanical Engineering, Mazandaran Institute of Technology, Babol, Iran \\ ${ }^{2}$ Department of Mechanical Engineering, Babol Noshiravni University of Technology, Babol, Iran
}

Received: 15 June 2017, Accepted: 8 September 2017

Published online: 10 April 2018.

\begin{abstract}
Nanofluid thermal radiation in a semi porous channel is investigated in existence of magnetic field. Influence of thermal radiation and Joule heating on energy equation is taken into account. Differential transform method is utilized to solve this problem. Effects of Reynolds number, Hartmann number, suction parameter, Eckert number, radiation parameter on flow and heat transfer are presented. Results show that temperature gradient augments with rise of Reynolds number, suction parameter, and Radiation parameter.
\end{abstract}

Keywords: Semi porous channel, nanofluid, magnetic field, single phase model, differential transform method.

\begin{tabular}{|l|l|l|l|}
\hline \multicolumn{4}{|c|}{ Nomenclature } \\
\hline$B_{0}$ & Magnetic induction & $\operatorname{Pr}$ & Prandtl number \\
\hline$R d$ & Radiation parameter & $q_{r}$ & Thermal radiation \\
\hline$H a$ & Hartmann number & $T$ & Fluid temperature \\
\hline$C_{p}$ & Specific heat capacity & $v, u$ & Vertical and horizontal velocities \\
\hline \multicolumn{5}{|c|}{ Greek symbols } \\
\hline$\sigma$ & Electrical conductivity & $\sigma_{e}$ & Stefan-Boltzmann constant \\
\hline$\eta$ & Similarity independent variable & $\alpha$ & Thermal diffusivity \\
\hline$\phi$ & Volume fraction of nanofluid & $\beta_{R}$ & mean absorption coefficient \\
\hline$\sigma$ & Surface tension & $\mu$ & Dynamic viscosity \\
\hline \multicolumn{5}{|l|}{ Subscripts } \\
\hline$s$ & Solid particles & $T$ & Thermal quantity \\
\hline$f$ & Base fluid & $\infty$ & for $\eta \rightarrow \infty$ \\
\hline$n f$ & nanofluid & 0 & at $\eta \rightarrow 0$ \\
\hline$C$ & Solutal quantity & & \\
\hline
\end{tabular}

\section{Introduction}

Nanofluid was proposed as innovative way to enhance heat transfer. Khan et al. [1] investigated nanofluid flow with slip motion influence in existence of inclined magnetic field. Bhatti et al. [2] studied the electric double layer effect on two phase flow in existence of magnetic field. Sheikholeslami and Ganji [3] presented various application of nanofluid in their review paper. Sheremet et al. [4] simulated the unsteady MHD flow in an enclosure. They used FDM to simulate 
that paper. Sheikholeslami and Ganji [5] investigated nanofluid flow inside a porous cavity by means of new numerical method. Ahmad and Mustafa [6] investigated the rotating nanofluid flow induced by an exponentially stretching. Their results revealed that temperature gradient reduces with augment of angular velocity. Sheikholeslami and Seyednezhad [7] investigated magnetic field effect on nanofluid heat transfer in a porous cavity. Sheikholeslami and Rokni [8] studied the nanofluid convective flow in a porous cylinder. Impact of magnetic nanofluid on film condensation was described by Heysiattalab et al. [9]. They concluded that $N u$ enhances with reducing size of nanoparticle.

Selimefendigil and Oztop [10] examined nanofluid conjugate conduction-convection mechanism in a titled cavity. They proved that temperature gradient augments with enhance of Grashof number. Sheikholeslami and Ganji [11] investigated nanofluid transportation in presence of external magnetic source. Sheikholeslami et al. [12] investigated nanofluid flow in a porous media in existence of magnetic field. Sheikholeslami et al. [13] utilized AGM for nanofluid flow between two pipes. Influence of non-uniform Lorentz forces on nanofluid flow style has been studied by Sheikholeslami Kandelousi [14]. He concluded that improvement in heat transfer reduces with rise of Kelvin forces. Sheikholeslami and Ganji [15] studied the electric field effect on nanofluid forced convection. Sheikholeslami and Ganji [16] demonstrated the effect of magnetic field on nanofluid natural convection. Sheikholeslami and Ganji [17] presented the nanofluid transportation in a curved cavity in presence of Lorentz forces. Several kinds of nanoparticles have been used by several researchers [18-24].

The aim of this article is to investigate impact of magnetic field on $\mathrm{CuO}$ - water nanofluid in existence thermal radiation. Similarity transformation is utilized to find the ordinary differential equations. Differential transform method is chosen to simulate this paper.

\section{Governing equation}

Steady nanofluid flow and heat transfer in a semi porous channel is considered. Fig. 1 shows the geometry and boundary conditions. Constant vertical magnetic field effect has been applied. Effects of thermal radiation and Joule heating on temperature distribution are taken into account. The lower plate is hot and the upper one is cold. Continuity, Navier stokes and energy equations are presented as follows:

$$
\begin{aligned}
& \frac{\partial u}{\partial x}+\frac{\partial v}{\partial y}=0 \\
& \rho_{\mathrm{nf}}\left(u \frac{\partial u}{\partial x}+v \frac{\partial u}{\partial y}\right)=-\frac{\partial p}{\partial x}+\mu_{n f}\left(\frac{\partial^{2} u}{\partial y^{2}}\right)-\sigma_{n f} B_{0}^{2} u \\
& \rho_{n f}\left(u \frac{\partial v}{\partial x}+v \frac{\partial v}{\partial y}\right)=-\frac{\partial p}{\partial y}+\mu_{n f}\left(\frac{\partial^{2} v}{\partial x^{2}}\right) \\
& \left(\rho C_{p}\right)_{n f}\left(u \frac{\partial T}{\partial x}+v \frac{\partial T}{\partial y}\right)=k_{n f}\left(\frac{\partial^{2} T}{\partial y^{2}}\right)+\sigma_{n f} B_{0}^{2} u^{2}-\frac{\partial q_{r}}{\partial y}
\end{aligned}
$$

where the radiation heat flux $q_{r}$ is considered according to Rosseland approximation such that $q_{r}=-\frac{4 \sigma_{e}}{3 \beta_{R}} \frac{\partial T^{4}}{\partial y}$ where $\sigma_{e}, \beta_{R}$ are the Stefan-Boltzmann constant and the mean absorption coefficient, respectively [27]. The fluid-phase temperature differences within the flow are assumed to be sufficiently small so that $T^{4}$ may be expressed as a linear function of temperature. This is done by expanding $T^{4}$ in a Taylor series about the temperature $T_{c}$ and neglecting higher order terms to yield, $T^{4} \cong 4 T_{c}^{3} T-3 T_{c}^{4}$. 
The effective density, effective heat capacity and electrical conductivity of the nanofluid are defined as:

$$
\begin{aligned}
\rho_{n f} & =(1-\phi) \rho_{f}+\phi \rho_{p}, \\
\left(\rho C_{p}\right)_{n f} & =(1-\phi)\left(\rho C_{p}\right)_{f}+\phi\left(\rho C_{p}\right)_{p}, \\
\frac{\sigma_{n f}}{\sigma_{f}} & =1+\frac{3\left(\frac{\sigma_{p}}{\sigma_{f}}-1\right) \phi}{\left(\frac{\sigma_{p}}{\sigma_{f}}+2\right)-\left(\frac{\sigma_{p}}{\sigma_{f}}-1\right) \phi} .
\end{aligned}
$$

The KKL (Koo-Kleinstreuer-Li) correlation has been utilized for viscosity of nanofluid

$$
\begin{aligned}
\mu_{e f f} & =\mu_{\text {static }}+\mu_{\text {Brownian }}=\mu_{\text {static }}+\frac{k_{\text {Brownian }}}{k_{f}} \times \frac{\mu_{f}}{\operatorname{Pr}_{f}} \\
k_{\text {Brownian }} & =5 \times 10^{4} \phi \rho_{f} c_{p, f} \sqrt{\frac{\kappa_{b} T}{\rho_{p} d_{p}}} g^{\prime}\left(T, \phi, d_{p}\right) \\
g^{\prime}\left(T, \phi, d_{p}\right) & =\left(a_{1}+a_{2} \ln \left(d_{p}\right)+a_{3} \ln (\phi)+a_{4} \ln (\phi) \ln \left(d_{p}\right)+a_{5} \ln \left(d_{p}\right)^{2}\right) \ln (T) \\
& +\left(a_{6}+a_{7} \ln \left(d_{p}\right)+a_{8} \ln (\phi)+a_{9} \ln (\phi) \ln \left(d_{p}\right)+a_{10} \ln \left(d_{p}\right)^{2}\right) .
\end{aligned}
$$

The related coefficient and properties of Cuo-water nanofluid is presented in table 1 and 2. Maxwell model and HamiltonCrosser model for irregular particle geometries by introducing a shape factor can be expressed as

$$
\frac{k_{n f}}{k_{f}}=\frac{k_{p}+(m+1) k_{f}-(m+1) \phi\left(k_{f}-k_{p}\right)}{k_{p}+(m+1) k_{f}+\phi\left(k_{f}-k_{p}\right)}
$$

in which $k_{p}$ and $k_{f}$ are the conductivities of the particle material and the base fluid. In this equation " $m$ " is shaper factor. Table 3 shows the different values of shape factors for various shapes of nanoparticles.

Boundary conditions are:

$$
\begin{aligned}
& u=b x, v=-v_{0}, T=T_{1} \quad \text { at } y=-a \\
& u=0, v=0, T=T_{2} \quad \text { at } y=+a
\end{aligned}
$$

where $b<0$ for shrinking walls channel and $b>0$ for stretching walls. Similarity transformation method has been utilized for obtain ordinary differential equations. The following non dimensional parameters are introduced:

$$
\eta=\frac{y}{a}, u=b x f^{\prime}(\eta), v=-a b f(\eta), \theta=\frac{T-T_{1}}{T_{1}-T_{2}}
$$

By using the above transformation, the final equations are obtained as follows:

$$
\begin{aligned}
& f^{i v}-H a^{2} \frac{A_{5}}{A_{2}} f^{\prime \prime}-R \frac{A_{1}}{A_{2}}\left(f^{\prime} f^{\prime \prime}-f f^{\prime \prime \prime}\right)=0 \\
& \left(1+\frac{4}{3 A_{4}} R d\right) \theta^{\prime \prime}+\operatorname{Pr} \frac{A_{3}}{A_{4}} f \theta^{\prime}+H a^{2} E c \frac{\operatorname{Pr}}{R} \frac{A_{5}}{A_{4}} f^{\prime 2}=0,
\end{aligned}
$$


where $R, H a, R d, E c$, Pr are stretching Reynolds number, Hartman number, Radiation parameter, Eckert number and Prandtl number. These constant parameters are defined as:

$$
\begin{aligned}
R & =\frac{a^{2} b}{v_{f}}, H a=B_{0} a \sqrt{\frac{\sigma_{f}}{\mu_{f}}}, R d=4 \sigma_{e} T_{c}^{3} /\left(\beta_{R} k_{f}\right), \\
E c & =\frac{\rho_{f}(b x)^{2}}{\left(\rho C_{p}\right)_{f} \Delta T}, \operatorname{Pr}=\frac{a^{2} b\left(\rho C_{p}\right)_{f}}{k_{f}}, \\
A_{1} & =\frac{\rho_{n f}}{\rho_{f}}, A_{2}=\frac{\mu_{n f}}{\mu_{f}}, A_{3}=\frac{\left(\rho C_{p}\right)_{n f}}{\left(\rho C_{p}\right)_{f}}, A_{4}=\frac{k_{n f}}{k_{f}}, A_{5}=\frac{\sigma_{n f}}{\sigma_{f}} .
\end{aligned}
$$

Moreover, boundary conditions becomes

$$
\begin{aligned}
& f^{\prime}(-1)=1, f^{\prime}(1)=0, \theta(-1)=1, \\
& f(-1)=\lambda=\frac{v_{0}}{a b}, f(1)=0, \theta(1)=0 .
\end{aligned}
$$

\section{Differential transform method (DTM)}

\subsection{Basic idea}

Basic definitions and operations of differential transformation are introduced as follows. Differential transformation of the function $f(\eta)$ is defined as follows:

$$
F(k)=\frac{1}{k !}\left[\frac{d^{k} f(\eta)}{d \eta^{k}}\right]_{\eta=\eta_{0}}
$$

In (14), $f(\eta)$ is the original function and $F(k)$ is the transformed function which is called the T-function (it is also called the spectrum of the $f(\eta)$ at $\eta=\eta_{0}$, in the $k$ domain). The differential inverse transformation of $F(k)$ is defined as:

$$
f(\eta)=\sum_{k=0}^{\infty} F(k)\left(\eta-\eta_{0}\right)^{k}
$$

by combining (14) and (15) $\mathrm{f}(\eta)$ can be obtained:

$$
f(\eta)=\sum_{k=0}^{\infty}\left[\frac{d^{k} f(\eta)}{d \eta^{k}}\right]_{\eta=\eta_{0}} \frac{\left(\eta-\eta_{0}\right)^{k}}{k !}
$$

Equation (16) implies that the concept of the differential transformation is derived from Taylor's series expansion, but the method does not evaluate the derivatives symbolically. However, relative derivatives are calculated by an iterative procedure that is described by the transformed equations of the original functions. From the definitions of (14) and (15), it is easily proven that the transformed functions comply with the basic mathematical operations shown in below. In real applications, the function $f(\eta)$ in (16) is expressed by a finite series and can be written as:

$$
f(\eta)=\sum_{k=0}^{N} F(k)\left(\eta-\eta_{0}\right)^{k}
$$


Equation (17) implies that $f(\eta)=\sum_{k=N+1}^{\infty}\left(F(k)\left(\eta-\eta_{0}\right)^{k}\right)$ is negligibly small, where $N$ is series size.

Theorems to be used in the transformation procedure, which can be evaluated from (14) and (15), are given below (Table4).

\subsection{Solution with differential transformation method}

Applying the differential transforms for Eqs. (10-11) gives:

$$
\begin{gathered}
(k+1)(k+2)(k+3)(k+4) F[k+4]-H a^{2} \frac{A_{5}}{A_{2}} f^{\prime \prime}(k+1)(k+2) F[k+2] \\
-R \frac{A_{1}}{A_{2}} \sum_{m=0}^{k}((m+1)(m+2) F[m+2](k-m+1) F[k-m+1]) \\
-R \frac{A_{1}}{A_{2}} \sum_{m=0}^{k}((m+1)(m+2)(m+3) F[m+3] F[k-m])=0 \\
\left(1+\frac{4}{3 A_{4}} \operatorname{Rd}\right)(k+2)(k+1) \Theta[k+2]+\operatorname{Pr} \frac{A_{3}}{A_{4}} \sum_{m=0}^{k}((m+1) \Theta[m+1] F[k-m]) \\
+H a^{2} E c \frac{\operatorname{Pr}}{R} \frac{A_{5}}{A_{4}} \sum_{m=0}^{k}((m+1) F[m+1](k-m+1) F[k-m+1])=0 \\
\Theta[0]=a_{2}, F[2]=a_{3}, F[3]=a_{4}, \Theta[1]=a_{6} .
\end{gathered}
$$

According to previous equations:

$$
\begin{aligned}
& F[0]=a_{1}, F[1]=a_{2}, F[2]=a_{3}, F[3]=a_{4}, \\
& F[4]=\frac{1}{12} H a^{2} \frac{A_{5}}{A_{2}} a_{3}+\frac{1}{12} R \frac{A_{1}}{A_{2}} a_{2} a_{3}-\frac{1}{4} R \frac{A_{1}}{A_{2}} a_{1} a_{4} \\
& F[5]=\frac{1}{20} H a^{2} \frac{A_{5}}{A_{2}} a_{4}+\frac{1}{30} R \frac{A_{1}}{A_{2}} a_{3}^{2}-\frac{1}{60} R \frac{A_{1}}{A_{2}} H a^{2} \frac{A_{5}}{A_{2}} a_{1} a_{3}+\frac{1}{20}\left(R \frac{A_{1}}{A_{2}}\right)^{2} a_{1}^{2} a_{4}, \ldots \\
& \Theta[0]=a_{5}, \Theta[1]=a_{6}, \Theta[2]=-1.5 \frac{\operatorname{Pr}}{R\left(3 A_{4}+4 R d\right)}\left(R A_{3} a_{1} a_{6}+H a^{2} A_{5} E c a_{2}^{2}\right) \\
& \Theta[3]=0.5 \frac{\operatorname{Pr}}{R\left(3 A_{4}+4 R d\right)^{2}}\left(-3 R A_{3} A_{1} a_{2} a_{6}-4 R A_{3} a_{2} a_{6} R d+3 a_{1}^{2} \operatorname{Pr} A_{3}^{2} a_{6} R+\right. \\
& \left.3 a_{1} \operatorname{Pr} H a^{2} E c A_{3} A_{5} a_{2}^{2}-12 H a^{2} E c a_{2} a_{3} A_{5} A_{4}-16 H a^{2} E c a_{2} a_{3} A_{5} R d\right), \ldots
\end{aligned}
$$


Finally we have:

$$
\begin{gathered}
F(\eta)=a_{1}+a_{2} \eta+a_{3} \eta^{2}+a_{4} \eta^{3}+\left(\frac{1}{12} H a^{2} \frac{A_{5}}{A_{2}} a_{3}+\frac{1}{12} R \frac{A_{1}}{A_{2}} a_{2} a_{3}-\frac{1}{4} R \frac{A_{1}}{A_{2}} a_{1} a_{4}\right) \eta^{4}+\ldots \\
\Theta(\eta)=a_{5}+a_{6} \eta+\left(-1.5 \frac{\operatorname{Pr}}{R\left(3 A_{4}+4 R d\right)}\left(R A_{3} a_{1} a_{6}+H a^{2} A_{5} E c a_{2}^{2}\right)\right) \eta^{2}+\ldots
\end{gathered}
$$

According to following equations and boundary conditions, $a_{1}, a_{2}, a_{3}, a_{4}, a_{5}, a_{6}$ can be obtained. By inserting obtained $a_{1}, a_{2}, a_{3}, a_{4}, a_{5}, a_{6}$ into Equations (24-25), it can be obtained the expression of $F(\eta)$ and $\Theta(\eta)$ which are transform of $f(\eta)$ and $\theta(\eta)$, respectively.

\section{Results and discussions}

Influence of thermal radiation on Magnetohydrodynamic CuO-water nanofluid flow and heat transfer in a semi porous channel with stretching wall is investigated. Influence of Joule heating on energy equation is taken into account. The ordinary differential equations are obtained by means of similarity transformation. Differential transform method (DTM) is chosen for solving final ODEs. Fig. 2 illustrates the verifications of presented result with those of obtained in previous works ([28] and [29]). As depicted in this figure, the current MAPLE code has good accuracy.

Influences of nanofluid volume fraction, shape of nanoparticles, Hartmann number, suction parameter, Eckert number, radiation parameter on velocity profile, temperature profile are presented in graphs and tables. Influences of shape of the nanoparticles on Nusselt number is shown in Table 5. Selecting Platelet leads to find the maximum Nusselt number. Therefore, Platelet nanoparticle has been selected for further investigation. Influence of volume fraction of nanofluid on temperature profile is depicted in Fig. 3. Temperature profile decreases with rise nanofluid volume fraction. So thermal boundary layer thickness has reverse relationship with nanofluid volume fraction. Effect of Reynolds number on velocity and temperature profiles is shown in Fig. 4. Vertical velocity and temperature profiles decreases with increase of Reynolds number. Horizontal velocity near the bottom wall decreases with increase of Reynolds number while opposite trend is observed near the upper wall. Fig. 5 exhibits the effect of Hartmann number on velocity and temperature profiles. Temperature profile enhances with increase of Hartmann number. Also velocity reduces with increase of Lorentz forces. Fig. 6 shows the effect of suction parameter on velocity and temperature profiles. Temperature and vertical velocity profiles enhances with rise of suction parameter. Horizontal velocity reduces with augment of suction parameter. Also the minimum point of velocity shift to lower wall.

\section{Conclusions}

Effect of magnetic field on forced and thermal radiation heat transfer in a channel is examined considering Joule heating effect. Roles of nanofluid volume fraction, shape of nanoparticles, Hartmann number, suction parameter, Eckert number, radiation parameter are discussed. As Hartman number and Eckert number increases, Nusselt number decreases while opposite trend is observed for skin friction coefficient. Also it can be found that Nusselt number increases with augment of Reynolds number, suction parameter, Radiation parameter. 


\section{Competing interests}

The authors declare that they have no competing interests.

\section{Authors' contributions}

All authors have contributed to all parts of the article. All authors read and approved the final manuscript.

\section{References}

[1] A.U. Khan, S. Nadeem, S.T. Hussain, Phase flow study of MHD nanofluid with slip effects on oscillatory oblique stagnation point flow in view of inclined magnetic field, Journal of Molecular Liquids 224 (2016) 1210-1219.

[2] M.M. Bhatti, A. Zeeshan, R. Ellahi, N. Ijaz, Heat and mass transfer of two-phase flow with Electric double layer effects induced due to peristaltic propulsion in the presence of transverse magnetic field, Journal of Molecular Liquids 230 (2017) 237-246.

[3] M. Sheikholeslami, D.D. Ganji, Nanofluid convective heat transfer using semi analytical and numerical approaches: A review, Journal of the Taiwan Institute of Chemical Engineers, 65 (2016) 43-77.

[4] Mikhail A. Sheremet, Ioan Pop, Natalia C. Roşca, Magnetic field effect on the unsteady natural convection in a wavy-walled cavity filled with a nanofluid: Buongiorno's mathematical model, Journal of the Taiwan Institute of Chemical Engineers 61 (2016) 211-222.

[5] M. Sheikholeslami, D.D. Ganji, Numerical modeling of magnetohydrodynamic $\mathrm{CuO}$-water transportation inside a porous cavity considering shape factor effect, Colloids and Surfaces A 529 (2017) 705-714.

[6] R. Ahmad, M. Mustafa, Model and comparative study for rotating flow of nanofluids due to convectively heated exponentially stretching sheet, Journal of Molecular Liquids, 220 (2016) 635-641.

[7] M. Sheikholeslami, M. Seyednezhad, Nanofluid heat transfer in a permeable enclosure in presence of variable magnetic field by means of CVFEM, International Journal of Heat and Mass Transfer, 114 (2017) 1169-1180.

[8] M. Sheikholeslami, Houman B. Rokni, Nanofluid convective heat transfer intensification in a porous circular cylinder, Nanofluid convective heat transfer intensification in a porous circular cylinder, Chemical Engineering \& Processing: Process Intensification, 120 (2017) 93-104.

[9] S. Heysiattalab, A. Malvandi, D.D. Ganji, Anisotropic behavior of magnetic nanofluids (MNFs) at filmwise condensation over a vertical plate in presence of a uniform variable-directional magnetic field, Journal of Molecular Liquids, 219 (2016) 875-882.

[10] Fatih Selimefendigil, Hakan F. Öztop, Conjugate natural convection in a cavity with a conductive partition and filled with different nanofluids on different sides of the partition, Journal of Molecular Liquids, 216 (2016) 67-77.

[11] M. Sheikholeslami, D.D. Ganji, Numerical analysis of nanofluid transportation in porous media under the influence of external magnetic source, Journal of Molecular Liquids, 233 (2017) 499-507.

[12] M. Sheikholeslami, Z. Ziabakhsh, D.D. Ganji, Transport of Magnetohydrodynamic nanofluid in a porous media, Colloids and Surfaces A: Physicochemical and Engineering Aspects, 520 (2017) 201-212.

[13] M. Sheikholeslami, M. Nimafar, D.D. Ganji, Nanofluid heat transfer between two pipes considering Brownian motion using AGM, Alexandria Engineering Journal (2017) 56, 277-283.

[14] Mohsen Sheikholeslami Kandelousi, Effect of spatially variable magnetic field on ferrofluid flow and heat transfer considering constant heat flux boundary condition, The European Physical Journal Plus (2014) 129- 248.

[15] M. Sheikholeslami, D.D. Ganji, Impact of electric field on nanofluid forced convection heat transfer with considering variable properties, Journal of Molecular Liquids 229 (2017) 566-573.

[16] M. Sheikholeslami, D.D. Ganji, Transportation of MHD nanofluid free convection in a porous semi annulus using numerical approach, Chemical Physics Letters 669 (2017) 202-210. 
[17] Mohsen Sheikholeslami, Davood Domiri Ganji, Numerical investigation of nanofluid transportation in a curved cavity in existence of magnetic source, Chemical Physics Letters, 667 (2017) 307-316.

[18] Inoue Shuhei, Iba Yushi, Matsumura Yukihiko. Drastic enhancement of effective thermal conductivity of a metal hydride packed bed by direct synthesis of single-walled carbon nanotubes. Int J Hydrogen Energy 2012;37:1836-41.

[19] M. Sheikholeslami, D.D. Ganji, Nanofluid hydrothermal behavior in existence of Lorentz forces considering Joule heating effect, Journal of Molecular Liquids 224 (2016) 526-537.

[20] M. Sheikholeslami, D.D. Ganji, M.M. Rashidi, Magnetic field effect on unsteady nanofluid flow and heat transfer using Buongiorno model, Journal of Magnetism and Magnetic Materials 416(2016)164-173.

[21] Kuma Jong Min, Yoo Seung Hwa, Ali Ghafar, Cho Sung Oh. Photocatalytic hydrogen production over CuO and TiO2 nanoparticles mixture. Int J Hydrogen Energy 2013;38:13541-6.

[22] Mohsen Sheikholeslami, Houman B. Rokni, Melting heat transfer influence on nanofluid flow inside a cavity in existence of magnetic field, International Journal of Heat and Mass Transfer 114 (2017) 517-526.

[23] Irnie Zakaria, W.H. Azmi, A.M.I. Mamat, Rizalman Mamat, R. Saidur, S.F. Abu Talib, W.A.N.W. Mohamed, Thermal analysis of Al2O3-water ethylene glycol mixture nanofluid for single PEM fuel cell cooling plate: An experimental study, International Journal of Hydrogen Energy, 41(2016) 5096-5112.

[24] Mohsen Sheikholeslami, Magnetic field influence on $\mathrm{CuO}-\mathrm{H} 2 \mathrm{O}$ nanofluid convective flow in a permeable cavity considering various shapes for nanoparticles, International Journal of Hydrogen Energy, 42 (2017) 19611-19621

[25] Raptis A. Radiation and free convection flow through a porous medium. Int. Commun. Heat Mass Transfer 1998; 25: 289-295.

[26] Misra, Shit, Rath, Flow and heat transfer of a MHD viscoelastic fluid in a channel with stretching walls: some applications to hemodynamics, Comput. Fluids (Int. J.) 37 (01) (2008) 1-11.

[27] K. Vajravelu, B.V.R. Kumar Analytic and numerical solutions of coupled nonlinear system arising in three-dimensional rotating flow. Int J Non-Linear Mech 39 (2004)13-24.

[28] A. Mehmood, A. Ali, Analytic solution of three-dimensional viscous flow and heat transfer over a stretching flat surface by Homotopy Analysis Method. ASME J Heat Trans. 2008 130: 12701-1 - 12701-7.

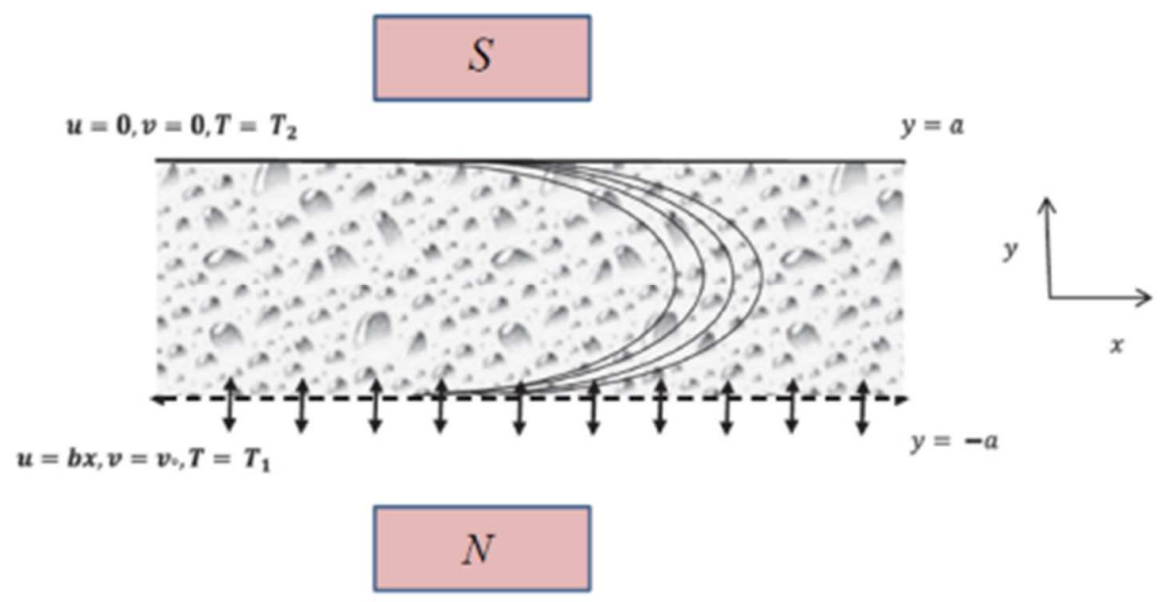

Fig. 1: Geometry of the problem 


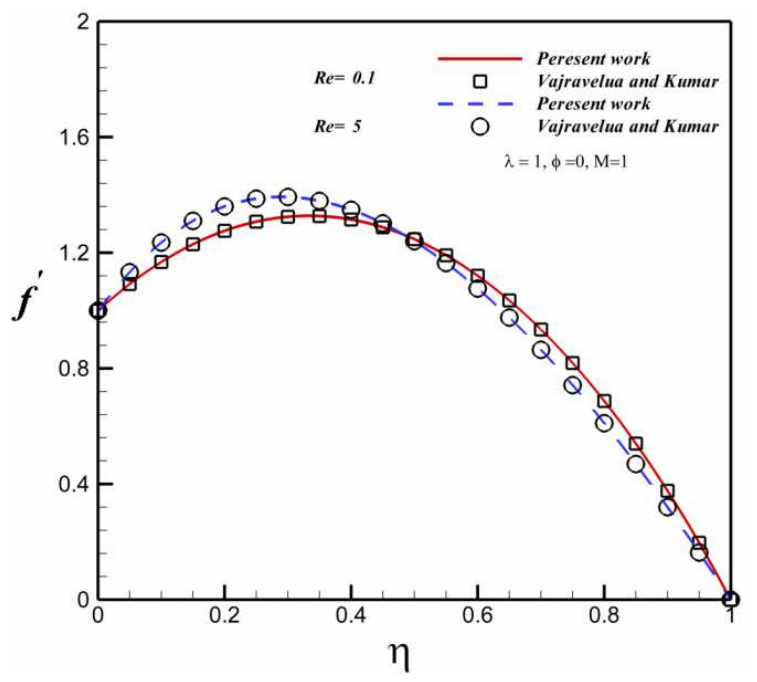

(a)

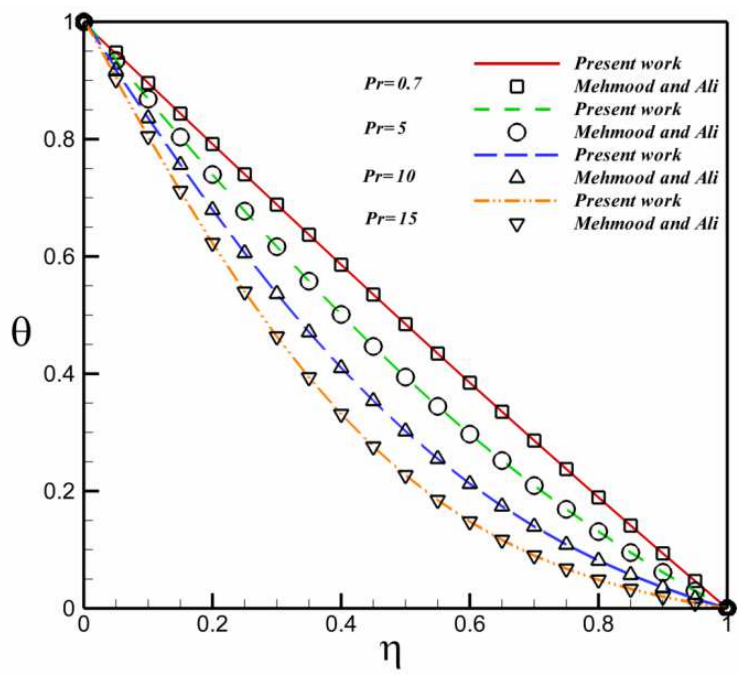

(b)

Fig. 2: Comparison of the velocity and temperature profiles between the present work and (a) [28]; (b) [29] for different values of $\operatorname{Pr}$ when $\lambda=0.5, M=1, \mathrm{R}=0.5$ and $K r=0.5$.

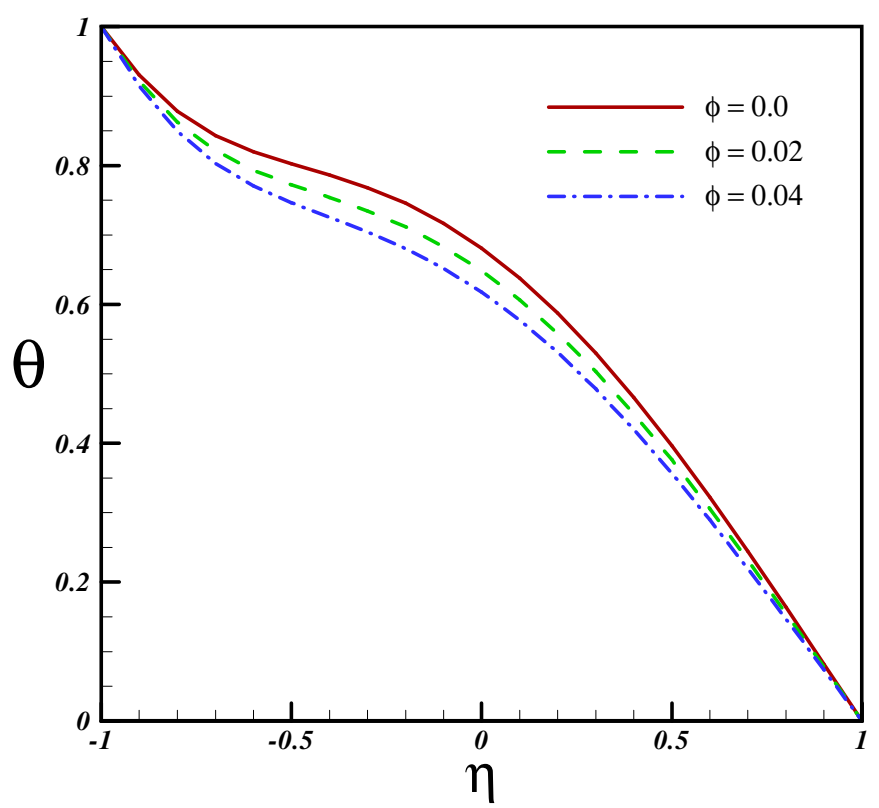

Fig. 3: Effect of nanofluid volume fraction on temperature profile when $R=1, H a=1, R d=0.5, \lambda=1, E c=0.5, m=$ 5.7. $\operatorname{Pr}=6.2$. 


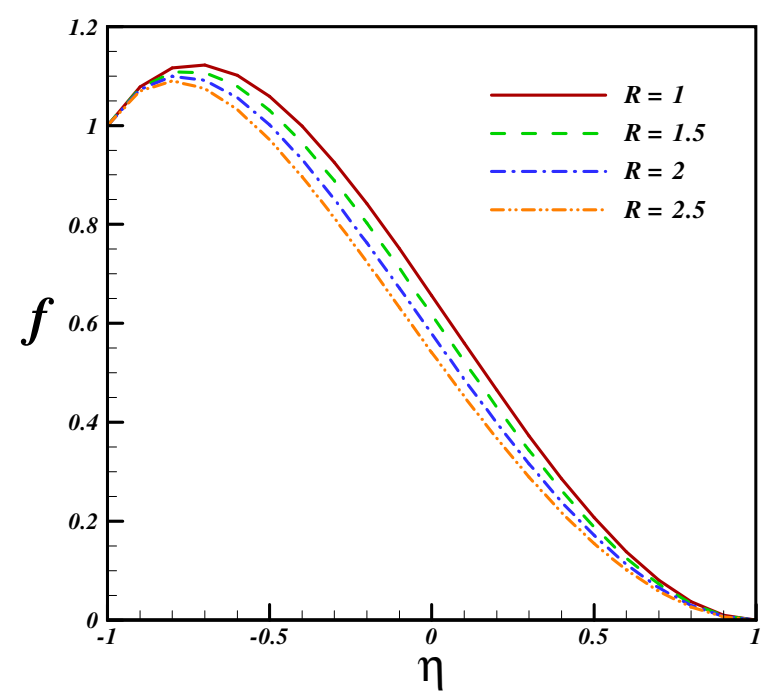

(a) $H a=1, \lambda=1, \phi=0.04$.

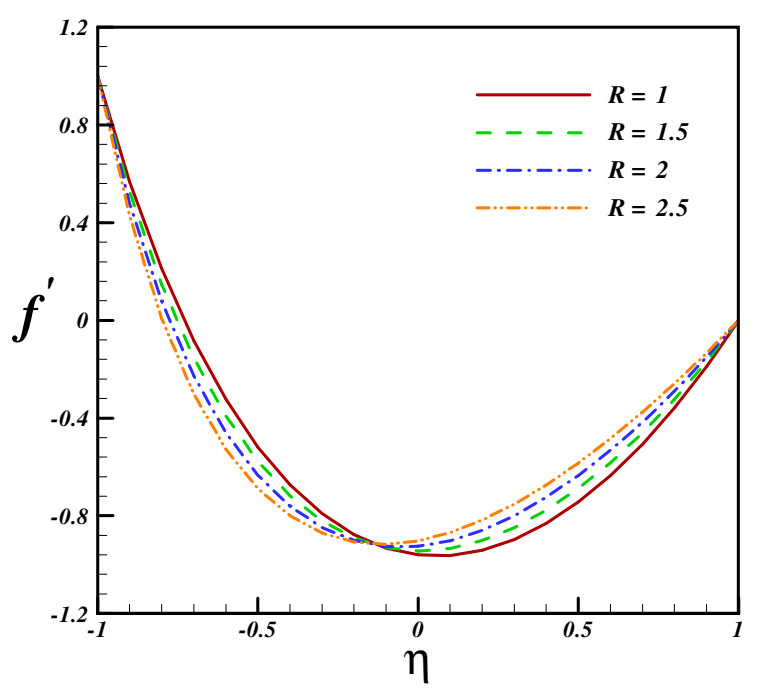

(b) $H a=1, \lambda=1, \phi=0.04$.

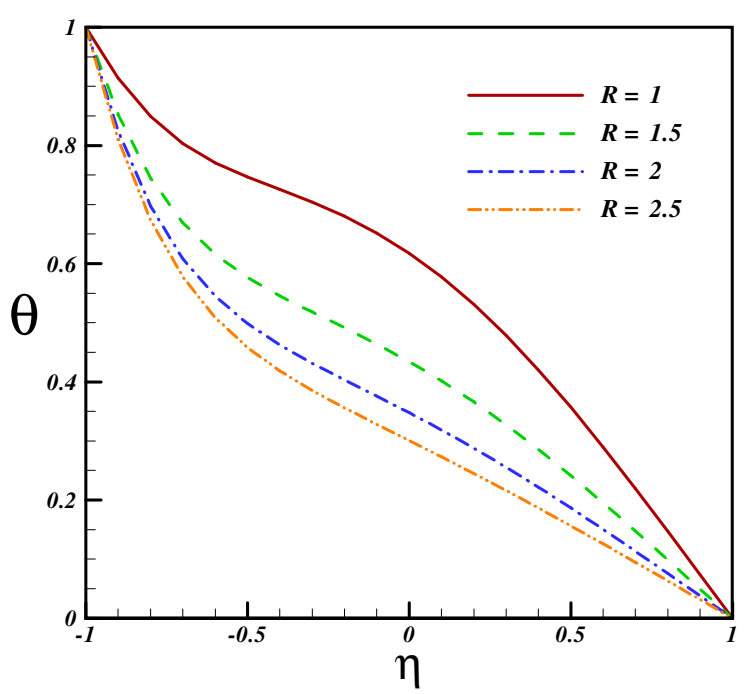

(c) $H a=1, R d=0.5, \lambda=1, E c=0.5, \phi=0.04, m=$ 5.7, $\operatorname{Pr}=6.2$.

Fig. 4: Effect of Reynolds number on velocity and temperature profiles. 


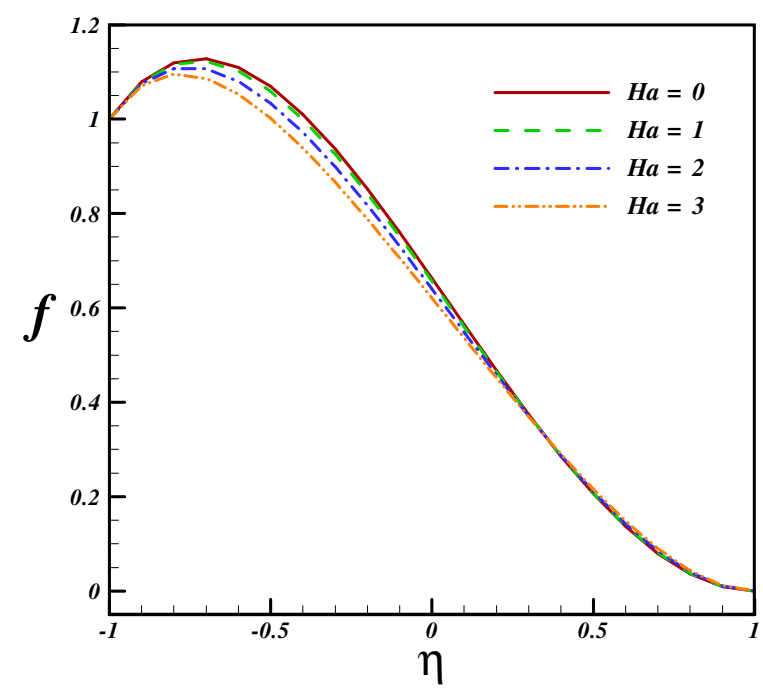

(a) $R=1, \lambda=1, \phi=0.04$.

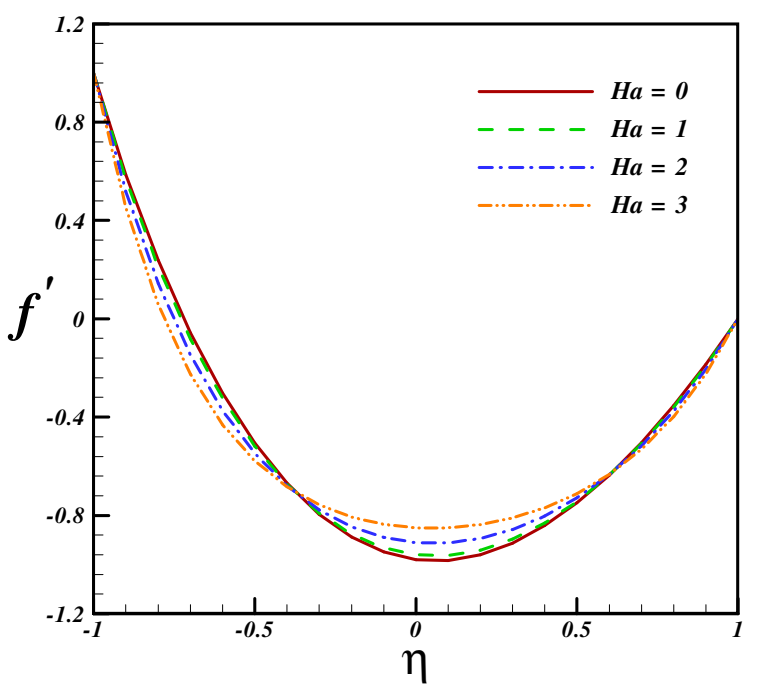

(b) $R=1, \lambda=1, \phi=0.04$.

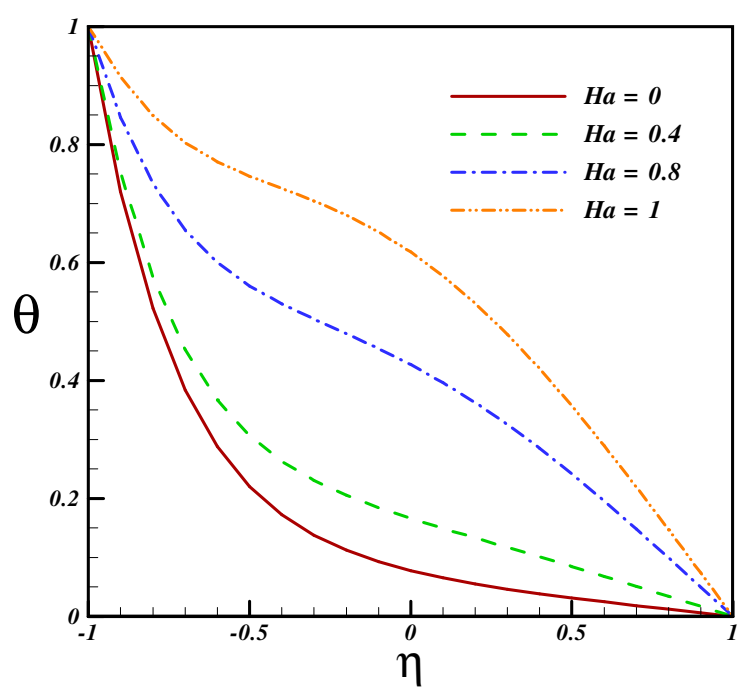

(c) $R=1, R d=0.5, \lambda=1, E c=0.5, \phi=0.04, m=5.7, \operatorname{Pr}=$ 6.2.

Fig. 5: Effect of Hartmann number on velocity and temperature profiles. 


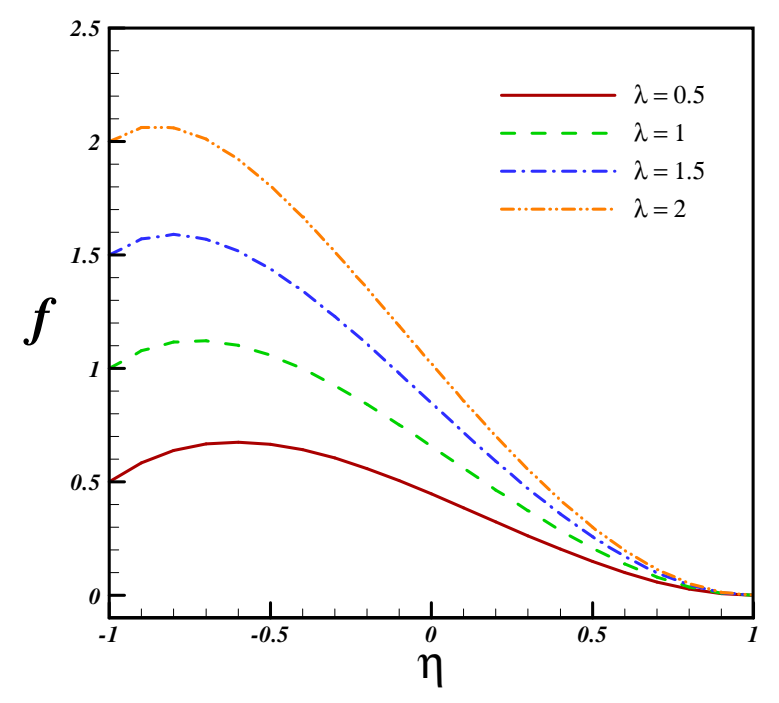

(a) $R=1, H a=1, \phi=0.04$.

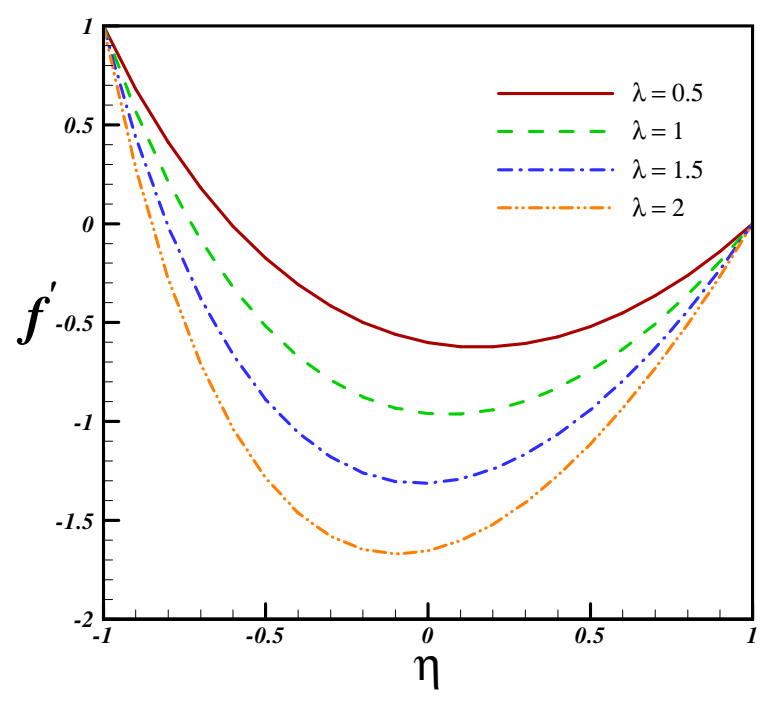

(b) $R=1, H a=1, \phi=0.04$.

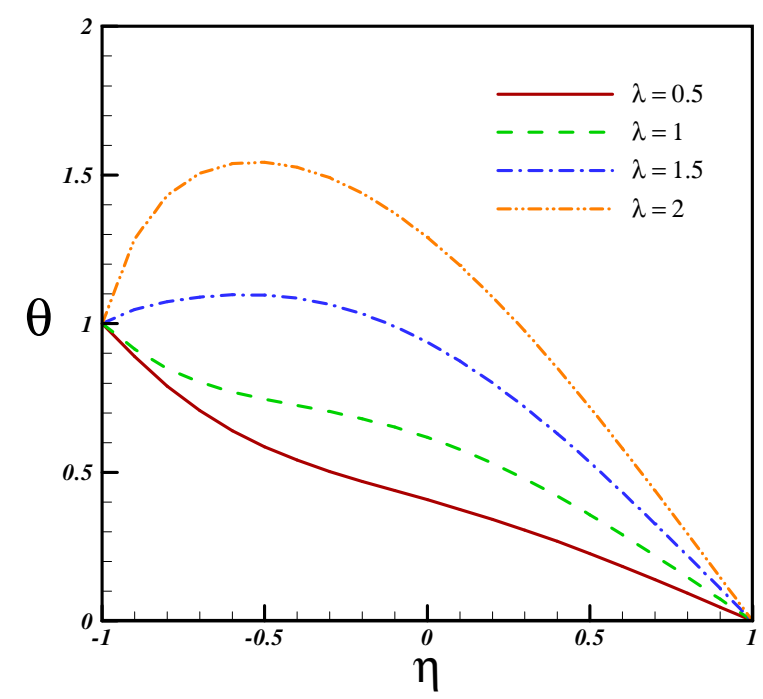

(c) $R=1, H a=1, R d=0.5, E c=0.5, \phi=0.04, m=$ 5.7, $\operatorname{Pr}=6.2$.

Fig. 6: Effect of suction parameter on velocity and temperature profiles.

Table 1: Thermo physical properties of water and nanoparticles.

\begin{tabular}{cccccc}
\hline & $\rho\left(\mathrm{kg} / \mathrm{m}^{3}\right)$ & $C_{p}(j / \mathrm{kgk})$ & $k(\mathrm{~W} / \mathrm{m} \cdot \mathrm{k})$ & $d_{p}(\mathrm{~nm})$ & $\sigma(\Omega \cdot \mathrm{m})^{-1}$ \\
\hline Pure water & 997.1 & 4179 & 0.613 & - & 0.05 \\
$\mathrm{CuO}$ & 6500 & 540 & 18 & 29 & $10^{-10}$ \\
\hline
\end{tabular}


Table 2: The coefficient values of $\mathrm{CuO}$ - Water nanofluids.

\begin{tabular}{cc}
\hline Coefficient values & CuO-Water \\
\hline$a_{1}-26.593310846$ \\
$a_{2}$ & -0.403818333 \\
$a_{3}$ & -33.3516805 \\
$a_{4}$ & -1.915825591 \\
$a_{5}$ & $6.42185846658 E-02$ \\
$a_{6}$ & 48.40336955 \\
$a_{7}$ & -9.787756683 \\
$a_{8}$ & 190.245610009 \\
$a_{9}$ & 10.9285386565 \\
$a_{10}$ & -0.72009983664 \\
\hline
\end{tabular}

Table 3: The values of shape factor of different shapes of nanoparticles.

Cylinder

Table 4: Some of the basic operations of Differential transformation method.

\begin{tabular}{ll}
\hline Original function & Transformed function \\
\hline$f(\eta)=\alpha g(\eta) \pm \beta h(\eta)$ & $F[k]=\alpha G[k] \pm \beta H[k]$ \\
$f(\eta)=\frac{d^{n} g(\eta)}{d \eta^{n}}$ & $F[k]=\frac{(k+n) !}{k !} G[k+n]$ \\
$f(\eta)=g(\eta) h(\eta)$ & $F[k]=\sum_{m-0}^{k} F[m] H[k-m]$ \\
$f(\tau)=\sin (\varpi \eta+\alpha)$ & $F[k]=\frac{\Phi^{k}}{k !} \sin \left(\frac{\pi k}{2}+\alpha\right)$ \\
$f(\tau)=\cos (\varpi \eta+\alpha)$ & $F[k]=\frac{\Phi^{k}}{k !} \cos \left(\frac{\pi k}{2}+\alpha\right)$ \\
$f(\eta)=e^{\lambda \eta}$ & $F[k]=\frac{\lambda^{k}}{k !}$ \\
$F(\eta)=(1+\eta)^{m}$ & $F[k]=\frac{m(m-1) \ldots(m-k+1)}{k !}$ \\
$f(\eta)=\eta^{m}$ & $F[k]=\delta(k-m)=\left\{\begin{array}{l}1, k=m \\
0, k \neq m\end{array}\right.$ \\
\hline
\end{tabular}


BISA S. M. Goudarzi and D.D. Ganji: Analytical investigation of thermal radiation effect on nanofluid forced...

Table 5: Nusselt number for various shape of the nanoparticles when $H a=1, R d=0.5, \lambda=1, E c=0.5, \phi=0.04, m=$ $5.7, \operatorname{Pr}=6.2$.

\begin{tabular}{ccccc}
\hline R & Spherical & Platelet & Cylinder & Brick \\
\hline 1 & 1.735301 & 1.815146 & 1.790366 & 1.757658 \\
1.5 & 3.21957 & 3.259504 & 3.247116 & 3.230759 \\
2 & 3.892636 & 3.91125 & 3.905465 & 3.897842 \\
2.5 & 4.236621 & 4.241594 & 4.240018 & 4.237987 \\
\hline
\end{tabular}

